\title{
CORRESPONDENCE
}

\section{Submitting a manuscript}

\section{To the Editors:}

I feel a little stupid. Recently, I wanted to submit a manuscript to the European Respiratory Journal (ERJ). I went to the ERJ website but, rather bafflingly, did not seem to be able to find a reference as to how one could submit. After Googling I found http://mc.manuscriptcentral.com/erj. But there the system immediately defeated me. I had to sign in with a name and password. Not being aware of having the required login details, I tried the Help button by providing my e-mail address, and then enjoyed the bliss of eternal silence. In all fairness, a message actually arrived after about 30 minutes, but I had given up all hope before that time.

Whilst I was waiting, I noticed that there was a facility to register, so that is what I did. But I was immediately stopped in my tracks because the system said it already knew me. I tried the Help button again by submitting my e-mail address, and received a password within a reasonable time, together with my first request. Now, though, the system would not let me submit a manuscript without filling in keywords and the like. I dislike systems that collect unnecessary information about individuals. I only want to submit a manuscript and there is no reason why a system should demand that I provide this information; I might do so if politely asked.

Eventually, the system said that all had been accomplished and that I could now submit a manuscript. Forget it, it just did not work. By this time I had spent an hour or more in futile activities. So, in desperation, I rebooted my computer and tried to establish contact again with Manuscript Central.

I have now submitted the manuscript. It took about 5 hours from the start. I had to copy information that was already provided in the manuscript, such as author names and affiliations. In a number of cases, I did not want to accept what the system forced upon me, such as "Cole, Time J" instead of "Cole, Tim J", and "rosenthal, mark", instead of "Rosenthal, Mark"; in addition, the latter's affiliation was omitted by the software. The system said that I was not allowed to change the information. I also had to copy from the manuscript the abstract text, the legends to the figures, etc. In the old days you printed the text, wrote a cover letter, put it in an envelope with address, licked a stamp and affixed it to the envelope, then put it in a mailbox. Maybe 15 minutes work. Manuscript Central seems to have made this process bureaucracy to the highest level of perfection whilst grinding the process down to the lowest possible pace; this may be a bureaucrat's delight but as an author, I dislike it. Ironically, if the manuscript is accepted I also have to pay for all my work.

I first used a computer in the 1970s and wrote my first software. I also run four (used to be five) websites, including those via which I provide people with free software, and I handle a large number of quite complicated pieces of software on a daily basis. So at first blush one would think that I have enough experience and insight to make submission of a manuscript a breeze. I am never too old to learn but it seems my talents are failing me. It must be Alzheimer's but I remember from a more lucid period of life that I thought that computers should never run our lives.

I am not writing this to upset anyone as I am convinced that everyone works to offer the best service. Most people would not dare submit a manuscript and then complain about the procedure. But I am a retired person, no longer involved in the rat race, and I have nothing to lose; only science that makes me tick. Of course, the senior author would normally leave this to a junior and never be aware of that junior's frustrations. Therefore, I thought that it might be worthwhile letting you know what I, acting as a junior author, went through.

\section{P. Quanjer}

Dept of Pulmonary Disease and Paediatrics, Erasmus Medical Centre, Rotterdam, the Netherlands.

Correspondence: P. Quanjer, Dept of Pulmonary Disease and Paediatrics, Erasmus Medical Centre, Rotterdam, the Netherlands. E-mail: pquanjer@xs4all.nl

Statement of Interest: None declared.

DOI: $10.1183 / 09031936.00164709$

\section{From the Editors:}

We thank Prof. Quanjer for sharing with us the difficulties he had when trying to electronically submit a manuscript at http:/ / mc.manuscriptcentral.com/erj.

We respectfully disagree with Prof. Quanjer in his statement that he would to pay for page charges if his manuscript is

accepted by the European Respiratory Journal (ERJ), as this is clearly not the policy of the Journal.

However, we agree that electronic procedures can be impersonal and sometimes frustrating, especially when websites or our computer, or both, do not react as rapidly as we expect due to hardware bugs, software anomalies, or simply a website's maintenance. 
We also agree that even when everything is functioning normally, computer workflow can sometimes lead to unexpected, and often surprising, situations. For example, Associate Editors of the ERJ must now assess the potential of all submitted papers for presentation as continuing medical education (CME)-accredited studies. In very simple terms, besides the three classical options, and their corresponding boxes indicating whether a paper should be accepted, revised or rejected, two new options have recently appeared on the ERJ Editors' dashboards asking them to indicate their choice about the CME qualifications of the manuscript in question. Failing (or forgetting) to indicate their choice by ticking the appropriate box blocks the system. One ERJ Associate Editor has recently pointed out to us that indicating whether a rejected paper should be considered for CME is certainly not logical as the answer to this question is obviously negative. Still, this Editor will have to tick one of the two available boxes for CME qualification, even when the choice applies to rejected papers (which will obviously not be qualified for CME as they have already been rejected). Failing to do so will prevent the Editor from validating his/her screen and finalising their decision.

This very simple example illustrates how big the gap can be between the human mind and computer software. The former can often be brilliantly mobile and flexible (a quality known by neuroscientists as "plasticity"), allowing clever deductions to be made and meaning unnecessary steps can be avoided. The latter is always heavily, and sometimes hopelessly, systematic and rigid (a property described by computer engineers as "reliability") to help their users avoid mistakes and oblivion. Human mood is rarely cold, being either fully overjoyed or exceedingly frustrated when experiencing successes or failures. The only heat we will ever feel from the computer might simply come from the overburdened electrical wires of a machine that has never been switched off. The list of contrasts between a scientist's mind and the computer system can be endless, and is certainly too long to be given in detail here. One thing we can almost be certain about relates to the sense of humour and humility that characterise mankind. We thank Prof. Quanjer for his sense of humour when sharing with us his challenging experience as a "retired junior" submitting author. As "unretired senior" scientists, we also feel frustrated seeing our young colleagues and our children working with computers much faster than we can do, but we are nonetheless grateful to these evil machines when we remember the amount of paperwork we did when submitting our manuscripts no longer than a decade ago. For the sake of our authors and Editorial Board, we humbly promise that we will try to improve the ERJ submission system to make life easier for all users, junior and senior colleagues alike.

However, we are not certain that computer machines will ever sense the joys or frustrations of their users. Quite honestly, we would be surprised and most worried if they do...

\section{A.T. Dinh-Xuan and V. Brusasco, ERJ Chief Editors on behalf of the ERJ Editorial Board}

Statement of Interest: Statements of interest for A.T. DinhXuan and V. Brusasco can be found at www.erj.ersjournals. $\mathrm{com} / \mathrm{misc} /$ statements.dtl

DOI: $10.1183 / 09031936.00004510$

\section{Pulmonary hypertension and lung diseases: a suggestion for revision of the clinical classification}

\section{To the Editors:}

We read with great interest the new clinical classification of pulmonary hypertension $(\mathrm{PH})[1]$, that represents a tremendous and successful effort to summarise the existing evidence of a rapidly evolving field in a physician-friendly manner. We want to focus on the inclusion of sarcoidosis, pulmonary Langerhans cells histiocytosis (PCLH) and lymphangioleiomyomatosis (LAM) in the subgroup of $\mathrm{PH}$ with unclear or multifactorial aetiologies (Group 5). Although raising such an issue might seem pedantic, we believe that there are good reasons for reconsidering the group place of these disorders.

Interstitial lung diseases (ILD) consist of disorders of known (e.g. collagen vascular disease, environmental, drug-induced) as well as unknown causes. The latter include idiopathic interstitial pneumonias, granulomatous lung disorders (e.g sarcoidosis), and other forms of ILDs, including LAM, PLCH and eosinophilic pneumonia [2].
Group 5.2 diseases may have a systemic component but their pulmonary manifestations far outnumber the manifestations of any other system. They mainly represent diffuse parenchymal lung diseases with distinctive and well-defined clinicopathological features that can affect all aspects of lung anatomy (interstitium, airspaces, peripheral airways and vessels), resulting in $\mathrm{PH}$ in a proportion of patients. Sarcoidosis presents with associated lung involvement in up to $95 \%$ of cases [3] and respiratory failure is the most common cause of death in Europe and the USA. Furthermore, mean pulmonary artery pressure has been significantly correlated with diffusion deficit and pulmonary vascular resistance with arterial oxygen tension in this setting [4]. PCLH is an ILD, preferentially affecting heavy smokers. LAM usually presents with cystic degeneration of the lungs and is often misdiagnosed initially as asthma or chronic obstructive pulmonary disease. It is a fact that the pathogenesis of $\mathrm{PH}$ for these disease entities is incompletely understood and may be multifactorial, but the same is true for all other ILDs [5]. Not surprisingly, no PAH-specific therapies have been approved for all these disease entities. 